\title{
Cognitive style, personality and vulnerability to postnatal depression
}

Lisa Jones, Jan Scott, Caroline Cooper, Liz Forty, Katherine Gordon Smith, Pak Sham, Anne Farmer, Peter McGuffin, Nick Craddock and lan Jones

\section{Background}

Only some women with recurrent major depressive disorder experience postnatal episodes. Personality and/or cognitive styles might increase the likelihood of experiencing postnatal depression.

\begin{abstract}
Aims
To establish whether personality and cognitive style predicts vulnerability to postnatal episodes over and above their known relationship to depression in general.

\section{Method}

We compared personality and cognitive style in women with recurrent major depressive disorder who had experienced one or more postnatal episodes (postnatal depression (PND) group, $n=143$ ) with healthy female controls (control group, $n=173$ ). We also examined parous women with recurrent major depressive disorder who experienced
\end{abstract}

no perinatal episodes (non-postnatal depression (NPND) group, $n=131$ ).

\section{Results}

The PND group had higher levels of neuroticism and dysfunctional beliefs, and lower self-esteem than the control group. However, there were no significant differences between the PND and NPND groups.

\section{Conclusions}

Established personality and cognitive vulnerabilities for depression were reported by women with a history of postnatal depression, but there was no evidence that any of these traits or styles confer a specific risk for the postnatal onset of episodes.

\section{Declaration of Interest}

None.
The link between traits such as personality and cognitive style and vulnerability to depression is well established. A range of measures have been robustly associated with depression including neuroticism, ${ }^{1}$ dysfunctional attitudes ${ }^{2}$ and low self-esteem. ${ }^{2}$ The link to depression occurring in relationship to childbirth has not been as extensively investigated and findings are less robust, but studies have reported evidence of association with neuroticism, ${ }^{3}$ introversion, ${ }^{3}$ perfectionism, ${ }^{4}$ low self-esteem, ${ }^{5}$ and dysfunctional cognitions. ${ }^{6,7}$ It remains to be established, however, whether measures of personality and/or cognitive style predict vulnerability to postnatal episodes over and above their known association with depression in general. Identifying specific vulnerability factors would have both theoretical and clinical importance. For example, in addition to giving important clues about aetiology, they could allow identification of women at high risk of postnatal depression and indicate those women in whom early intervention may be of benefit. They may also influence the development of psychotherapies specific to the postpartum context, and may predict adherence with, and response to, other treatment approaches such as medication.

In this study we address two questions: (1) does personality and/or cognitive style differ in women with a history of postnatal depression compared with healthy controls; and (2) do measures of personality and/or cognitive style distinguish women with a history of postnatal depression from women with recurrent major depressive disorder who have not experienced perinatal episodes.

\section{Method}

\section{Samples}

Two samples are included in this study: (1) a patient sample (parous women with major recurrent depression, who were not in an episode of depression when recruited and assessed, divided into two subsamples of (a) having a history of postnatal depression, and (b) not having an episode of depression in relation to childbirth); and (2) a healthy female control sample.

The patient sample was recruited as part of our ongoing research programme to investigate genetic and non-genetic determinants of major affective disorders, which uses systematic and non-systematic recruitment methods. Patient records of community mental health teams (CMHTs) across the UK are systematically screened to identify patients with major affective disorders, and, at the discretion of the responsible medical officer, all patients deemed suitable for inclusion are invited to participate. Invitations are sent to patients only when, in the opinion of the CMHT, they do not currently meet diagnostic criteria for a major depressive episode. Additionally, patient volunteers are recruited non-systematically via advertisements for participants with affective disorders in local general practitioner surgeries, local media and via patient support organisations (Depression Alliance and MDF The BiPolar Organisation). Patients are included in our research programme if they meet the following criteria: capable of giving voluntary informed written consent; aged 18 years or over; meet DSM-IV, ICD- $10^{9}$ and Research Diagnostic Criteria (RDC) ${ }^{10}$ for a major affective disorder; and, because of the fact that they are recruited for molecular genetic studies, UK/Ireland White ethnicity. Patients are excluded if they: have only experienced affective illness in relation to, or as a consequence of, alcohol or substance misuse or dependence; have only experienced affective illness as a consequence of medical illness or medication; or are an intravenous drug user with a lifetime diagnosis of dependency. Across our whole research programme, $44 \%$ of suitable patients identified via CMHTs have refused to participate and we have been unable to make contact with a further $14 \%$.

To be included in the study reported here, patients must also be parous women meeting DSM-IV, ${ }^{8} \mathrm{ICD}-10^{9}$ and $\mathrm{RDC}^{10}$ criteria 
for major recurrent depressive disorder. Additional exclusion criteria are: an organic brain disorder or other cognitive problem that impedes ability to complete questionnaires; or biologically related to another study participant. Our final eligible sample for the study reported here is 379 parous women with recurrent major depressive disorder, with $39.3 \% \quad(n=149)$ recruited systematically.

The control participants are a subsample of participants who had originally been recruited to the GENESiS (Genetic Environmental-Nature of Emotional States in Siblings) study. ${ }^{11}$ This study recruited over 34000 individuals through general practices in England and Wales. Index participants were individuals registered with these practices in the age range 18-55 years who had no current serious medical illness or disability. Participants included as controls in our research programme had scores on the Sham Composite Index of liability to depression and anxiety $(' G \text { ' })^{11}$ that were in the lowest $20 \%$ of the GENESiS population. ' $G$ ' comprises responses to the following self-report measures: General Health Questionnaire (12-item version; GHQ-12); ${ }^{12}$ short form of the Neuroticism subscale from the Eysenck Personality Questionnaire (EPQ-N); ${ }^{13}$ and two subscales from the Mood and Anxiety Symptoms Questionnaire (MASQ), ${ }^{14}$ which measure levels of anxious arousal (MASQ-AA) and high positive affect (MASQ-HPA). Participants selected for our programme were additionally screened using a standardised semi-structured telephone interview administered by trained psychiatrists or psychologists. None had a personal or family history of psychiatric illness (defined as having consulted a healthcare professional, or having received a diagnosis or treatment from a healthcare professional for a mental health problem). Of the control participants in our programme, there were 173 women who comprised the healthy control sample for this study.

\section{Psychiatric assessments}

Patients in our research programme are interviewed face to face, usually in their own homes, by trained psychiatrists or psychologists using the Schedules for Clinical Assessment in Neuropsychiatry $(\mathrm{SCAN})^{15}$ and psychiatric/general practice case notes are reviewed. These data are combined for each participant to form a written case vignette. Best-estimate lifetime diagnoses are made according to DSM-IV, ICD-10 and RDC. The vignettes are also used to rate other key clinical variables (including age at onset, number of illness episodes, history of psychosis, number of psychiatric admissions) and women are asked pregnancy by pregnancy about episodes of depression occurring in relationship to childbirth. Although a complete SCAN interview is not carried out for each perinatal episode, questions are asked about depressive symptoms to establish whether the episode met DSM-IV criteria for major depression and to establish the approximate week of onset preor post-delivery. Each patient is diagnosed, and has key clinical variables including postnatal episode status rated independently by at least two members of the research team and consensus is reached. Interrater reliability is high. This has been formally assessed using 20 cases and resulted in mean kappa statistics of $0.85,0.83$ and 0.80 for DSM-IV, ICD-10 and RDC diagnoses respectively. Mean kappa statistics for other key clinical variables (categorical) ranged from 0.81 to 0.99 . Mean intraclass correlation coefficients for other key clinical variables (continuous) ranged from 0.91 to 0.97 .

\section{Definition of postnatal episodes}

Only parous women, and therefore those who had an opportunity to experience an episode of postnatal depression, were included in the analysis. An episode of postnatal depression was defined as an episode of DSM-IV major depression with onset within 6 weeks of delivery (PND group) - corresponding to our recent finding that familiality of postnatal depression is maximised between 6 and 8 weeks. ${ }^{16}$ A wider definition of perinatal depressive episode was employed in defining the group of women who had not suffered with an episode of postnatal depression. Women in the nonpostnatal depression group (NPND group) had given birth but had not experienced the onset of a major depressive episode either during their pregnancy or within 6 months of delivery. The PND group comprised 143 women and the NPND group comprised 131 women. The remaining 105 parous women in our sample were excluded from further analyses as they had an episode onset either during pregnancy or between 6 weeks and 6 months after delivery. Figure 1 summarises the women included in the patient groups.

\section{Questionnaires}

The following self-rated questionnaires, which all have demonstrated validity and reliability, were part of a larger pack of questionnaires administered to both patients and controls.

\section{Rosenberg Self-Esteem Questionnaire (SEQ) ${ }^{17}$}

The SEQ measures trait self-esteem. It is a 10 -item questionnaire comprising a positive and a negative subscale. Scores range from 5 to 20 on both subscales with high scores on the positive subscale reflecting high positive self-esteem and high scores on the negative subscale reflecting low negative self-esteem. Combining these subscales produces a total SEQ score (range 10-40), with higher total scores reflecting higher total self-esteem.

\section{Dysfunctional Attitudes Scale (DAS) ${ }^{18}$}

The DAS measures underlying beliefs and attitudes. We used the 24-item version of the DAS. As well as a total score (range 24-168), the DAS gives three subscale scores (achievement, dependency and self-control) each ranging from 8 to 56 . Higher scores indicate higher levels of dysfunctional attitudes.

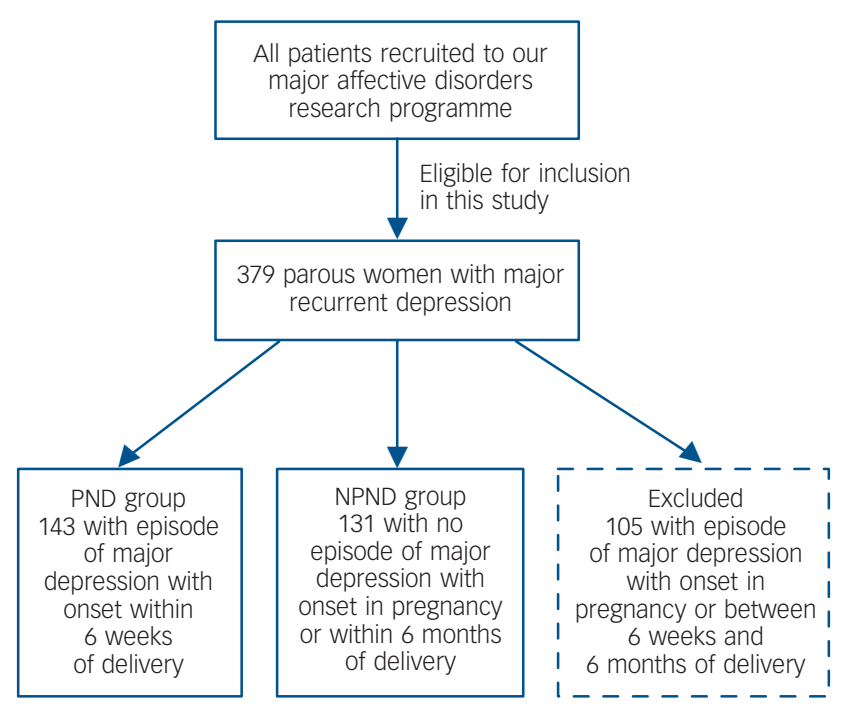

Fig. 1 Summary of participants included in the patient groups PND, postnatal depression; NPND, non-postnatal depression. 


\section{Eysenck Personality Questionnaire (EPQ) $)^{13}$}

We used the 90-item version of the EPQ. The EPQ gives scores for three personality dimensions: extraversion, neuroticism and psychoticism. Extraversion scores range from 0 to 21, neuroticism scores range from 0 to 23 , and psychoticism scores range from 0 to 25 .

\section{Beck Depression Inventory $(\mathrm{BDI})^{19}$}

The BDI assesses the presence and/or severity of current depressive symptoms. It comprises 21 items and total scores range from 0 to 63 .

\section{Procedure}

The procedure for systematically recruited patients and volunteer patients was identical. After the semi-structured interview, the questionnaires were left with patients to rate and return to the team (stamped, addressed return envelopes were provided). They were given written instructions to complete all of the questionnaires at the same time within 1 week of receiving them. If the questionnaires were not returned after 1 month, a reminder letter was sent out, along with another copy of the questionnaires and return envelope. If the questionnaires were still not returned after a further 2 weeks, a reminder telephone call was made.

The control participants received the questionnaires via post following their telephone interview. Otherwise, the procedure was identical to that outlined above.

This study received all necessary national research ethics service approval.

\section{Statistical analysis}

The three groups (PND group, NPND group and control group) were compared for age using one-way analysis of variance (ANOVA), followed by post hoc comparisons using the Tukey Test. Differences in key clinical variables between the PND and NPND groups were examined using independent samples $t$-tests or chi-squared tests.

The distributions of the questionnaire scores in each of the groups approximated to normal, thus the groups were compared on the BDI, and all cognitive style and personality measures using univariate one-way ANOVAs, followed by the Tukey Test. Oneway univariate analyses of covariance (ANCOVA) were then carried out with possible confounders (age, BDI scores) included as covariates. The PND and NPND groups were compared using one-way ANCOVA with age, BDI scores and all clinical variables that differed between the two groups as covariates.

All analyses were undertaken using SPSS (version 14.0) for Windows.

\section{Results}

\section{Sample characteristics}

As shown in Table 1, the NPND group was significantly older (mean age 53 years) than both the PND and control groups (mean age 47 and 49 years respectively). The two patient groups scored significantly higher on the BDI (measuring current levels of depressive symptomatology) than the control group (mean BDI scores of 17,17 and 3 respectively).

Table 2 summarises the clinical features of the patient groups. The PND and NPND groups were well matched on number of episodes of depression, number of psychiatric hospital admissions, number of deliveries, and history of psychosis. The NPND group had a significantly older age at illness onset (mean age 30 years),

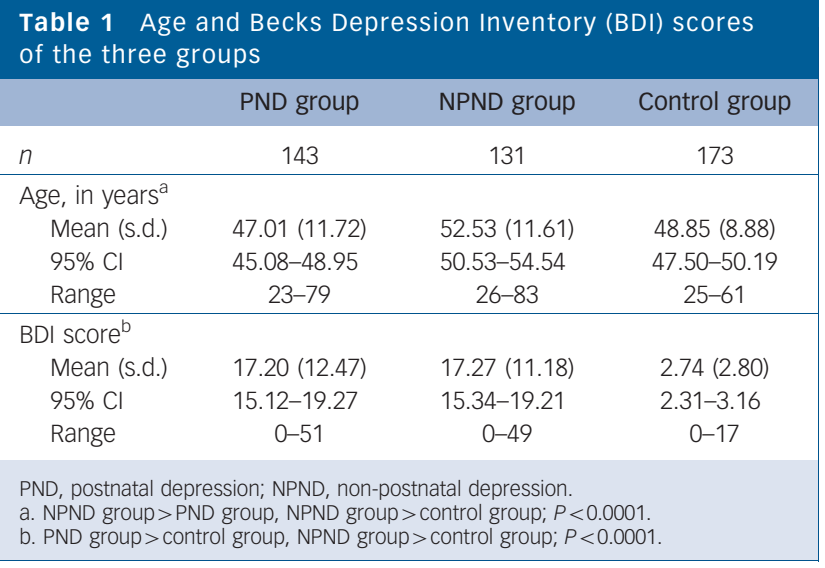

\begin{tabular}{|c|c|c|}
\hline & PND group & NPND group \\
\hline$n$ & 143 & 131 \\
\hline \multicolumn{3}{|c|}{ Number of episodes of depression } \\
\hline Mean (s.d.) & $4.97(2.61)$ & $4.38(2.65)$ \\
\hline $95 \% \mathrm{Cl}$ & $4.48-5.35$ & $3.91-4.84$ \\
\hline Range & $2-16$ & $2-15$ \\
\hline \multicolumn{3}{|c|}{ Age at illness onset, years ${ }^{a}$} \\
\hline Mean (s.d.) & $23.07(6.61)$ & $30.03(11.40)$ \\
\hline $95 \% \mathrm{Cl}$ & $21.93-24.21$ & 23.04-32.03 \\
\hline Range & $11-39$ & $10-61$ \\
\hline \multicolumn{3}{|c|}{ History of psychotic symptoms, $n$ (\%) } \\
\hline Never present & $124(86.7)$ & $119(90.8)$ \\
\hline Present & $15(10.5)$ & $12(9.2)$ \\
\hline Not known & $4(2.8)$ & - \\
\hline \multicolumn{3}{|c|}{ Number of admissions } \\
\hline Mean (s.d.) & $0.79(1.20)$ & $0.81(1.58)$ \\
\hline $95 \% \mathrm{Cl}$ & $0.57-0.98$ & $0.51-1.06$ \\
\hline Median (IQR) & $0.00(1.00)$ & $0.00(1.00)$ \\
\hline Range & $0-6$ & $0-10$ \\
\hline \multicolumn{3}{|c|}{ Number of deliveries } \\
\hline Mean (s.d.) & $2.46(1.06)$ & $2.26(1.02)$ \\
\hline $95 \% \mathrm{Cl}$ & $2.27-2.65$ & $2.09-2.44$ \\
\hline Range & $1-6$ & $1-6$ \\
\hline
\end{tabular}

defined as age at first clinically significant functional impairment due to affective illness, than the PND group (mean age 23 years).

\section{Comparison of personality and cognitive style between the groups}

Mean scores for each of the groups on each of the personality and cognitive style measures are presented in Table 3. Compared with the control group, the PND group showed significantly lower levels of self-esteem and extraversion, and significantly more dysfunctional attitudes, neuroticism and psychoticism. There were no significant differences on any of the measures between the PND and NPND groups.

These data were further analysed using ANCOVA. The three groups were compared on all measures controlling for age and BDI scores. The inclusion of the covariates did not alter the findings. The PND and NPND groups were compared controlling for age, BDI scores and the clinical variable that differed between the two groups, age at illness onset. Again, the inclusion of the covariates did not alter the findings. 


\begin{tabular}{|c|c|c|c|}
\hline & PND group & NPND group & Control group \\
\hline \multicolumn{4}{|c|}{$\begin{array}{l}\text { Rosenberg Self-Esteem } \\
\text { Questionnaire }\end{array}$} \\
\hline$n$ & 132 & 128 & 170 \\
\hline \multicolumn{4}{|l|}{ Total score ${ }^{a}$} \\
\hline Mean (s.d.) & $23.99(5.97)$ & $24.92(6.31)$ & $34.18(3.66)$ \\
\hline $95 \% \mathrm{Cl}$ & $22.96-25.02$ & $23.82-26.03$ & 33.63-34.74 \\
\hline \multicolumn{4}{|c|}{ Positive subscale } \\
\hline Mean (s.d.) & $12.74(2.96)$ & $13.05(3.09)$ & $16.92(3.73)$ \\
\hline $95 \% \mathrm{Cl}$ & $12.23-13.25$ & $12.51-13.59$ & $16.63-17.22$ \\
\hline \multicolumn{4}{|c|}{ Negative subscale ${ }^{a}$} \\
\hline Mean (s.d.) & $11.25(3.56)$ & $11.88(3.80)$ & $17.26(2.39)$ \\
\hline $95 \% \mathrm{Cl}$ & $10.64-11.86$ & $11.21-12.54$ & $16.90-17.62$ \\
\hline \multicolumn{4}{|c|}{ Dysfunctional Attitudes } \\
\hline \multicolumn{4}{|l|}{ scale } \\
\hline$n$ & 133 & 129 & 170 \\
\hline \multicolumn{4}{|l|}{ Total score ${ }^{b}$} \\
\hline Mean (s.d.) & $98.18(24.90)$ & $93.74(29.01)$ & $71.92(17.25)$ \\
\hline $95 \% \mathrm{Cl}$ & 93.91-102.45 & 88.69-98.80 & $69.31-74.53$ \\
\hline \multicolumn{4}{|l|}{ Achievement $^{\mathrm{b}}$} \\
\hline Mean (s.d.) & 31.69 (11.44) & 30.09 (12.33) & $19.51(7.97)$ \\
\hline $95 \% \mathrm{Cl}$ & $29.73-33.65$ & $27.94-32.23$ & $18.30-20.71$ \\
\hline \multicolumn{4}{|l|}{ Dependency ${ }^{b}$} \\
\hline Mean (s.d.) & $35.72(9.07)$ & $33.56(10.46)$ & $25.52(7.08)$ \\
\hline $95 \% \mathrm{Cl}$ & $34.17-37.28$ & $31.74-35.38$ & $24.45-26.59$ \\
\hline \multicolumn{4}{|l|}{ Self-contro| $\left.\right|^{b}$} \\
\hline Mean (s.d.) & 30.77 (8.24) & $30.10(9.64)$ & $26.89(7.65)$ \\
\hline $95 \% \mathrm{Cl}$ & $29.35-32.18$ & $28.42-31.78$ & 25.74-28.05 \\
\hline \multicolumn{4}{|c|}{$\begin{array}{l}\text { Eysenck Personality } \\
\text { Ouestionnaire }\end{array}$} \\
\hline$n$ & 141 & 130 & 173 \\
\hline \multicolumn{4}{|l|}{ Extraversion ${ }^{\mathrm{a}}$} \\
\hline Mean (s.d.) & $9.13(5.58)$ & $9.16(5.30)$ & $14.83(4.91)$ \\
\hline $95 \% \mathrm{Cl}$ & $8.21-10.06$ & $8.24-10.08$ & $14.10-15.57$ \\
\hline \multicolumn{4}{|l|}{ Neuroticism } \\
\hline Mean (s.d.) & $17.04(4.84)$ & $16.98(4.88)$ & $5.14(3.67)$ \\
\hline $95 \% \mathrm{Cl}$ & $16.24-17.85$ & $16.14-17.83$ & $4.59-5.69$ \\
\hline \multicolumn{4}{|l|}{ Psychoticism $^{\text {b }}$} \\
\hline Mean (s.d.) & $3.30(2.50)$ & $2.92(2.18)$ & $2.02(2.07)$ \\
\hline $95 \% \mathrm{Cl}$ & $2.89-3.72$ & $2.54-3.29$ & $1.71-2.33$ \\
\hline
\end{tabular}

\section{Discussion}

\section{Postnatal mood disorders}

Mood disorders occurring in relationship to childbirth are an important public health issue with suicide now a leading cause of maternal death in the UK. ${ }^{20}$ Postnatal or postpartum depression are terms that have gained widespread use over the past 40 years and the concept has undoubtedly had positive effects in reducing stigma and in the development of clinical services for women who become ill at this time. However, the scientific validity of the concept of postnatal depression has been questioned over the past decade as evidence emerged to suggest that childbirth is not associated with increased rates of depression ${ }^{21-23}$ and that in clinical presentation and response to treatment, postnatal depression does not differ from depression occurring at other times. ${ }^{24}$ Nevertheless, findings are inconsistent and the literature is far from clear.

Other studies have suggested that in a subgroup of women who experience postnatal depression there is a specific association between childbirth and the onset of depression ${ }^{25}$ and that the postnatal period may indeed be a period of increased risk for depression when analyses take account of other known risk factors for depression. ${ }^{26,27}$ In addition, we have recently demonstrated that a vulnerability to postnatal triggering is familial and therefore potentially influenced by genetic factors. ${ }^{16}$ If some women have a particular vulnerability to postpartum episodes of depression, identifying putative risk factors for postnatal depression will increase the clinician's ability to prospectively identify those at high risk and also contribute to research on stress-vulnerability models of mood disorders.

Studies that have been carried out so far suggest considerable overlap between the risk factors for postnatal depression and for depression in general. ${ }^{28}$ However, it is important that studies determine what factors may specifically increase an individual's susceptibility to postnatal episodes - be they biological or psychosocial.

\section{Personality and cognitive style}

One line of research that has proved very productive in increasing understanding of stress-vulnerability models of depression is the study of premorbid traits. There is robust evidence that personality traits, especially high premorbid levels of neuroticism ${ }^{1}$ and abnormalities of cognitive style such as stable negative selfesteem $^{2,29}$ are associated with vulnerability to depression. We examined whether personality and/or cognitive style predict vulnerability to postnatal episodes of depression as it is plausible that the transition to motherhood is a particular challenge for some women (e.g. those with low self-esteem). ${ }^{6}$

Our findings suggest that women with a history of major depression associated with childbirth demonstrate characteristic depressogenic profiles with lower self-esteem (greater negative evaluation of self, and less positive evaluation of self), higher levels of dysfunctional attitudes (greater need for achievement, greater dependency on others, and greater need for control of self), and higher levels of neuroticism compared with healthy controls. These findings are consistent with previous studies of women with postnatal depression and of women who experience recurrent (non-postnatal) depression compared with healthy controls. ${ }^{3-5,7}$

The present study then extended the analysis to explore whether any of these personality and cognitive measures has a specific association with postnatal depression over and above the robustly demonstrated associations with vulnerability to depression in general. We did not find any unique cognitive or personality characteristics that differentiated between women with recurrent major depressive disorder who did or did not have a history of postnatal depression. This suggests that future studies of postnatal depression should incorporate this three-group design to distinguish between factors that influence the depression diathesis in general and the puerperal onset in particular. Study designs that do not include a non-postnatal depression group and simply demonstrate differences between women with postnatal depression and healthy controls cannot demonstrate specificity for depression triggered by childbirth and erroneous conclusions may result.

\section{Strengths}

Our samples were large and post hoc power calculations, undertaken using nQuery Advisor (version 4.0) for Windows, showed that on all of the questionnaire measures our sample size had $>99 \%$ power to detect at the 0.05 level the difference in means between the three groups. The patients in this study were: narrowly defined - they met lifetime diagnostic criteria according to three widely-used psychiatric classification systems; well characterised - illness data were collected using a validated 
semi-structured interview and case-note review, and a range of clinical variables, including puerperal status, were rated using consensus method, with excellent interrater reliability; and representative - a large percentage were systematically ascertained. The control group was unaffected and selected for a low risk of developing a mood disorder.

\section{Limitations}

Our results must be interpreted in light of a number of limitations. First, this is a cross-sectional study so we cannot determine whether the differences in personality and cognitive style observed in the women with depression are a cause of recurrent depressive episodes or the psychological sequelae of this experience. For example, elevated neuroticism scores in those with recurrent depression may represent trait vulnerability ${ }^{30,31}$ or merely reflect residual symptoms. ${ }^{32}$ Prospective longitudinal studies are necessary if we are to determine whether there are any true differences between factors that influence a general depression diathesis and puerperal triggers in particular. For example, future studies of postnatal depression would ideally undertake cognitive and personality ratings from at least early pregnancy until up to a year postnatally and compare these with clinical outcomes.

We excluded women with onset of depression antenatally or between 6 weeks and 6 months after delivery to ensure our PND and NPND groups were as clean as possible. We chose the cut-off of 6 weeks after delivery for illness onset for the PND group on the basis of our own published work that familiality of postnatal depression is maximised when onset is between 6 and 8 weeks postpartum. ${ }^{16}$ A sensitivity analysis using later cut-offs might be most illuminating, although larger sample sizes are required to do this while retaining adequate statistical power. Consideration of women with antenatal onset of depression would be interesting and important, and future work should aim to explore this prospectively in a large sample of women with antenatal depression.

Furthermore, we have investigated only limited aspects of personality and cognitive style. There are a number of other measures such as coping styles, attributions, self-representations, attachment styles, novelty-seeking traits, affective temperaments and perfectionism, which may reveal differences between women vulnerable to postnatal triggering and those with no perinatal episodes of depression. It would also be important to incorporate measures that rate beliefs specifically related to motherhood ${ }^{6}$ rather than relying solely on general measures of cognitive style. Similarly, future work may benefit from including measures of social factors (e.g. relationship with partner) that may differentiate sensitivity to postnatal depression from sensitivity to depression at other times in life.

Other limitations need to be noted. For example, we have included patients recruited both systematically (via CMHTs) and opportunistically (via advertisements). Analyses repeated using only the former group, however, reveal an unchanged pattern of findings. There was a high refusal/non-response rate within our systematically ascertained group, which might have introduced responder bias. However, there was no difference in response rate between the PND and NPND groups, and any bias between patients and controls is likely to be conservative assuming that patients with higher levels of depressogenic cognitive style were those less likely to participate. Also, to maximise our power to detect differences, we used a control group selected for low risk of developing a mood disorder, so-called 'supernormal' controls. The measures of cognitive style examined here, however, were not used to define the control group, and it is unlikely that the dif- ferences we have found are accounted for merely by the selection of controls.

The link with childbirth is a challenge to our understanding of the aetiology of depression and has received very different explanations by those from divergent theoretical backgrounds. Biological explanations that implicate the massive hormonal fluctuations at this time vie with psychosocial explanations that emphasise difficulties in the role transition associated with motherhood. The perinatal period is a complex time with immense changes at the biological, psychological and social level, and it is therefore difficult to differentiate which factors in each of these domains play the most significant role in the onset of depressive episodes at this time. Our findings, however, suggest that personality and cognitive style alone do not identify women with a specific postnatal vulnerability.

\footnotetext{
Lisa Jones, PhD, Department of Psychiatry, School of Clinical and Experimental Medicine, University of Birmingham, UK; Jan Scott, FRCPsych, Department of Psychiatry, Institute of Neuroscience, University of Newcastle, UK; Caroline Cooper MSC, Department of Psychiatry, School of Clinical and Experimental Medicine, University of Birmingham, UK; Liz Forty, PhD, Department of Psychological Medicine Medical School, Cardiff University, UK; Katherine Gordon Smith, PhD, Department of Psychiatry, School of Clinical and Experimental Medicine, University of Birmingham, and Department of Psychological Medicine, Medical School, Cardiff University, UK; Pak Sham, MRCPsych, Department of Psychiatry, LKS Faculty of Medicine, University of Hong Kong, China; Anne Farmer, FRCPsych, Peter McGuffin, FRCPsych, SGDP Research Centre, Institute of Psychiatry, UK. Nick Craddock, FRCPSych, Ian Jones, MRCP ch, Departent of Psychological Medicine, Medical Schol, Cardiff Universit, MRCPSych, Department of Psychological Medicine, Medical School, Cardiff University,
UK

Correspondence: Dr lan Jones, Senior Department of Psychological Medicine The Henry Wellcome Building for Biomedical Research in Wales, Academic Avenue, Cardiff University, Heath Park, Cardiff CF14 4XN, UK. Email: JonesIR1@cf.ac.uk

First received 6 Feb 2009, final revision 26 Oct 2009, accepted 2 Dec 2009
}

\section{Funding}

We are very grateful to the Medical Research Council for financial support. This work was supported by UK Medical Research Council grants G0000647 and G9700821.

\section{Acknowledgements}

We are indebted to all the mental health professionals who helped us to recruit patients for this work. We thank all the participants who so generously gave us their time and support.

\section{References}

1 Martin M. Neuroticism as a predisposition towards depression: a cognitive mechanism. Pers Ind Diff 1985; 6: 353-65

2 Jones L, Scott J, Haque S, Gordon-Smith K, Heron J, Caesar S, et al. Cognitive style in bipolar disorder. $\mathrm{Br} J$ Psychiatry 2005; 187: 431-7.

3 Verkerk GJ, Denollet J, Van Heck GL, Van Son MJ, Pop VJ. Personality factors as determinants of depression in postpartum women: a prospective 1-year follow-up study. Psychosom Med 2005; 67: 632-7.

4 Mazzeo SE, Slof-Op't Landt MC, Jones I, Mitchell K, Kendler KS, Neale MC, et al. Associations among postpartum depression, eating disorders, and perfectionism in a population-based sample of adult women. Int J Eat Disord 2006; 39: 202-11.

5 Beck CT. Predictors of postpartum depression: an update. Nurs Res 2001; $\mathbf{5 0}$ : 275-85.

6 Moorhead S, Owens J, Scott J. Developing and piloting of the Pregnancy Related Beliefs Questionnaire (PRBQ). Behav Cogn Psychother 2003; 31: 207-13.

7 Church NF, Brechman-Toussaint ML, Hine DW. Do dysfunctional cognitions mediate the relationship between risk factors and postnatal depression symptomatology? J Affect Disord 2005; 87: 65-72.

8 American Psychiatric Association. Diagnostic and Statistical Manual of Mental Disorders (Fourth Edition, Text Revision) (DSM-IV-TR). APA, 2000.

9 World Health Organization. The International Classification of Diseases 10 Classification of Mental and Behavioural Disorders. Diagnostic Criteria for Research. WHO, 1993. 
10 Spitzer RL, Endicott J, Robins E. Research diagnostic criteria: rationale and reliability. Arch Gen Psychiatry 1978; 35: 773-82.

11 Sham PC, Sterne A, Purcell S, Cherny S, Webster M, Rijsdijk F, et al. GENESiS creating a composite index of the vulnerability to anxiety and depression in a community-based sample of siblings. Twin Res 2000; 3: 316-22.

12 Goldberg DP, Gater R, Sartorius N, Ustun TB, Piccinelli M, Gureje O, et al. The validity of two versions of the GHQ in the WHO study of mental illness in general health care. Psychol Med 1997; 27: 191-7.

13 Eysenck HJ, Eysenck SBG. Manual of the Eysenck Personality Questionnaire. Hodder \& Stoughton, 1975.

14 Watson D, Clark LA, Weber K, Assenheimer JS, Strauss ME, McCormick RA Testing a tripartite model: II. Exploring the symptom structure of anxiety and depression in student, adult, and patient samples. J Abnorm Psychol 1995; 104: 15-25.

15 Wing JK, Babor T, Brugha T, Burke J, Cooper JE, Giel R, et al. SCAN. Schedules for Clinical Assessment in Neuropsychiatry. Arch Gen Psychiatry 1990; 47: 589-93.

16 Forty L, Jones L, Macgregor S, Caesar S, Cooper C, Hough A, et al. Familiality of postpartum depression in unipolar disorder: results of a family study. Am J Psychiatry 2006; 163: 1549-53.

17 Rosenberg M. Society and the Adolescent Self-Image. Princeton University Press, 1965.

18 Weissman A, Beck A. Development and validation of the Dysfunctional Attitudes Scale. Dissertation Abstracts, 1978

19 Beck AT, Steer RA. The Beck Depression Inventory. Harcourt Brace Jovanovich, 1987.

20 Jones I, Craddock N. Bipolar disorder and childbirth: the importance of recognising risk. $\mathrm{Br} J$ Psychiatry 2005; 186: 453-4.

21 O'Hara MW, Zekoski EM, Philipps LH, Wright EJ. Controlled prospective study of postpartum mood disorders: comparison of childbearing and nonchildbearing women. J Abnorm Psychol 1990; 99: 3-15.
22 Cooper PJ, Campbell EA, Day A, Kennerley H, Bond A. Non-psychotic psychiatric disorder after childbirth. A prospective study of prevalence, incidence, course and nature. Br J Psychiatry 1988; 152: 799-806.

23 Cox JL, Murray D, Chapman G. A controlled study of the onset, duration and prevalence of postnatal depression. Br J Psychiatry 1993; 163: 27-31.

24 Cooper C, Jones L, Dunn E, Forty L, Haque S, Oyebode F, et al. Clinical presentation of postnatal and non-postnatal depressive episodes. Psychol Med 2007; 37: 1273-80.

25 Cooper P, Murray L. Prediction, detection, and treatment of postnatal depression. Arch Dis Child 1997; 77: 97-9.

26 Eberhard-Gran M, Eskild A, Tambs K, Samuelsen SO, Opjordsmoen S. Depression in postpartum and non-postpartum women: prevalence and risk factors. Acta Psychiatr Scand 2002; 106: 426-33.

27 Munk-Olsen T, Laursen T, Pedersen C, Mors O, Mortensen P. New parents and mental disorders: a population-based register study. J Am Med Assoc 2006; 296: 2582-9.

28 O'Hara MW, Swain AM. Rates and risk of postpartum depression - a metanalysis. Int Rev Psychiatry 1996; 8: 37-54.

29 Scott J, Stanton B, Garland A, Ferrier IN. Cognitive vulnerability in patients with bipolar disorder. Psychol Med 2000; 30: 467-72.

30 Duggan C, Sham P, Lee A, Minne C, Murray R. Neuroticism: a vulnerability marker for depression evidence from a family study. J Affect Disord 1995; 35: 139-43.

31 Fanous A, Gardner CO, Prescott CA, Cancro R, Kendler KS. Neuroticism, major depression and gender: a population-based twin study. Psychol Med 2002; 32: 719-28

32 Farmer A, Redman K, Harris T, Mahmood A, Sadler S, Pickering A, et al. Neuroticism, extraversion, life events and depression. The Cardiff Depression Study. Br J Psychiatry 2002; 181: 118-22.

\section{Poems by doctors}

\section{Junkie on the Phone}

\section{Kirsten Emmott}

You don't have a headache.

The GP you named doesn't know you.

The pharmacist recognizes your name.

You even called me before.

I won't prescribe the drugs.

Play the game elsewhere.

Call up some other doctor.

Set out your lies:

"Doctor, here is my lie.

I want you to join me in my lying.

Pretend I am sick.

Give me what will make me sicker.

Give me a stick

with which to beat myself.

Help me to die."

Kirsten Emmott was born in Edmonton, Alberta. She studied medicine at the University of British Columbia in Vancouver and practises in Comox, British Columbia. She published a collection of poems, How Do You Feel? (Soho Nis Press); some of her poetry can also be found on www.kirstenemmott.com. This poem is from The Naked Physician: Poems about the Lived of Patients and Doctors, edited by

R. Charach (Quarry Press). Reproduced with kind permission of the author.

Chosen by Femi Oyebode. 